\title{
Cross Layer Design for Efficient Video Streaming over LTE Using Scalable Video Coding
}

\author{
Rakesh Radhakrishnan, Balaaji Tirouvengadam, Amiya Nayak \\ School of Electrical Engineering and Computer Science, \\ University of Ottawa, 800 King Edward Ave, \\ Ottawa, Canada K1N 6N5 \\ E-mail: \{rradh015, btiro072, nayak\}@uottawa.ca
}

Received: August 1, 2012 Accepted: November 9, 2012 Published: December 16, 2012

DOI: $10.5296 / n p a . v 4 i 4.2171$

URL: http://dx.doi.org/10.5296/npa.v4i4.2171

\begin{abstract}
Third Generation Partnership Project (3GPP) Long Term Evolution (LTE) offers high data rate capabilities to mobile users, and network operators are trying to deliver a true mobile broadband experience over LTE networks. Mobile TV and Video on Demand (VoD) are expected to be the main revenue generators in the near future, and efficient video streaming over wireless is the key to achieve this goal. Video services use both unicast and multicast video transmission based on its applications. Enhanced Multimedia Broadcast/Multicast Service (EMBMS) is defined in 3GPP specification to support download delivery and streaming delivery to group users in LTE mobile networks. In 3GPP Release 8 specification, the EMBMS transmission is classified into single-cell transmission and MBSFN (Multicast Broadcast Single Frequency Network) transmission. In this paper, we propose a Scalable Video Coding (SVC) based video streaming scheme with dynamic adaptations and a scheduling scheme based on channel quality for unicast and multicast video transmissions. In our proposed unicast scheme, Channel Quality Indicator (CQI) feedbacks from User Equipment's (UE) are used for dynamic adaptations and scheduling. Cross layer signaling between Medium Access Control (MAC) and Real Time Transport (RTP) protocols is used to achieve the channel dependent adaptation for unicast in video server. Simulation results for unicast indicate improved video quality for more number of users with reduced bit rate video traffic. Approximately $13 \%$ video quality gain is observed for users at the cell edge using this adaptation scheme. We have also proposed a scheduling scheme for multicast over MBSFN networks. This scheme optimizes the radio spectrum allocation by adaptive modulation and coding (AMC) and frequency scheduling based on distribution of users in different channel quality regions. Through simulations we demonstrate that spectrum savings in the order of 72 to $82 \%$ is achievable in different user distribution scenarios with our proposed scheme.
\end{abstract}


Keywords: Adaptive Modulation and Coding, bandwidth adaptation, CQI feedback, cross layer design, H.264, LTE, MBSFN, multicast, SVC, unicast.

\section{Introduction}

Video streaming over wireless is expected to be one of the main revenue generators for current and future mobile broadband networks. Compared to other data services like web browsing, video streaming over wireless networks is challenging due to high bandwidth requirement and delay sensitive nature of video. In the recent past, ITU has selected LTEAdvanced and Wireless MAN-Advanced (WIMAX-Advanced) as Fourth Generation (4G) mobile broadband technologies [1]. Since LTE is evolved from 3G networks, many operators have chosen LTE as a pre-4G network option. Quality of Experience (QoE) similar to fixed broadband networks is expected to be delivered over these mobile technologies. However, the delivery of multimedia services over next generation mobile networks faces some unique challenges when compared to existing wired networks. The actual quality of reception conditions varies throughout the network, and heterogeneous receivers need to be supported. Therefore, the video streaming framework should be highly adaptive to mobile device capability and network conditions.

H.264/AVC (Advanced Video Coding) is one of the video codecs defined in 3GPP Release 6, and Multimedia Broadcast Multicast Service (MBMS) recommends only H.264 baseline profile. However, the SVC extension of H.264 allows efficient temporal, spatial and quality scalabilities. These scalabilities can be used for video bit stream adaptation based on user and network capabilities. A lot of research has been done on SVC based video transmission over wireless access networks, including LTE. Channel qualities of individual users are available in MAC layer of eNodeB (evolved NodeB, Base Station in an LTE system) in form of CQI feedbacks. Some of the LTE MAC frequency domain scheduling schemes presented in literature uses these feedbacks for channel dependent scheduling. Active dropping of video frames in MAC layer of WIMAX network based on Channel State Information (CSI) is discussed in [2]. Packet adaptation or scheduling in the MAC layer of base station is used by most of the existing works in literature. A cross-layer signaling framework for a dynamic scalable video adaptation in varying network capacity is presented in [3]. The authors compare a fast MAC-layer packet adaptation with a relatively slow and long-term adaptation in the application layer using a real H.264/SVC video. The authors conclude that dropping of packets in video server is a more efficient solution than MAC layer dropping in base station to reduce the congestion in both wired medium in the core network and wireless medium to the UE.

Mobile TV, games and other multimedia services will be the major contributors to the global mobile entertainment market according to a new report [4]. Multicast/broadcast plays a very important role in these entertainment services and applications. To address these new services, 3GPP has defined a Multimedia Broadcast/Multicast Service (MBMS) for UMTS in its Release 6 specification, in which the existing architecture is extended by the introduction of an MBMS bearer service and MBMS user service. 3GPP has specified a more advanced and Enhanced MBMS (EMBMS) service, which provides higher frequency efficiency and more reliable pointto-multipoint transmission for LTE. In 3GPP Release 8 specification, the EMBMS transmission 
is classified into single-cell transmission and MBSFN (Multicast Broadcast Single Frequency Network transmission). In MBSFN operation, MBMS data are transmitted simultaneously over the air from multiple tightly time synchronized cells. A group of those cells which are targeted to receive these data constitute the so-called MBSFN area [5]. Since the MBSFN transmission greatly enhances the Signal to Interference Noise Ratio (SINR), MBSFN transmission mode leads to significant improvement in spectral efficiency (SE) compared to single-cell MBMS service. This is extremely beneficial at the cell edge, where transmissions from neighboring cells (which are considered as interference in single-cell MBMS) are combined into useful signal energy, and hence the received signal strength is increased while, at the same time, the interference power is largely reduced.

In point-to-multipoint mode of MBMS, a group of MBMS subscribers listen to a common channel. They share same time and frequency resources as well as same Modulation and Coding Scheme (MCS). This implies that in order to fulfill Quality of Service (QoS) requirements, MCS has to be adjusted to the weakest terminal of a subscription group. So, adapting the MCS scheme to the weakest terminal is very important for satisfying the cell edge users. SVC provides an attractive option for sending same video in multiple layers as base layer and enhancement layers. Base layer reception is enough for basic quality of the video and adding of enhancement layers provide enhanced video quality. This can be used in single-cell MBMS service to provide basic quality to cell edge or low channel quality users and to provide high quality videos to high channel quality users. Some of the previous papers $[6,7]$ present options of sending one video stream with multiple modulations and coding schemes in cellular networks. However, authors use multicasting in single cell networks and MCS decisions are not optimized based on user distribution.

Channel dependent adaptations in unicast are usually done in the MAC layer because of the unavailability of channel state information in the video server. To overcome this limitation, we propose a cross layer signaling between MAC layer in the eNodeB and RTP layer in the video server. Average CQI values are signaled from MAC layer to RTP layer, and this information is used for adaptation by the RTP layer. Scalability features of scalable video are used for efficient adaptation in the video server. To exploit the frequency diversity of the channel, channel dependent MAC scheduling is used in the MAC layer. Adaptive GBR selection using CQI of the users is also combined with this scheme to improve the coverage of the cell by admitting more number of users in the cell. Part of this work appears in [26]. We also propose an AMC and scheduling scheme for multicast video traffic based on MBSFN channel quality measurements to increase video quality of users while maintaining same coverage as single layer video. We further enhance our scheme to increase the spectral efficiency using channel quality distribution information. Unlike existing SVC video transmission schemes over wireless, 3 layers of video are split into 3 sub-channels in the physical layer. This gives more control for physical layer adaptation and scheduling of each video layer separately.

Simulations conducted using OPNET modeler [8] and SVC video traces prove the benefits of proposed adaptation schemes for unicast and multicast. In the unicast adaptation scheme, the packet loss ratio is decreased and objective video quality is increased for all the users in cell coverage area. Increase of video quality for users in cell edge is also highly noticeable. This improved video quality comes with less bit rate video stream and corresponding throughput savings in wired and wireless medium from video server to the users. Our adaptive GBR 
selection scheme has the benefit of admitting more number of users and achieving more coverage using the same amount of radio resources. Approximately $13 \%$ video quality gain is observed for users at the cell edge using this adaptation scheme. In the proposed multicast scheme, coverage of low channel quality users is ensured using low MCS value for base layer video and higher video quality is provided to the users with good channel conditions using SVC quality enhancement layers. Through simulations we demonstrate that spectrum savings in the order of 72 to $82 \%$ is achievable in different user distribution scenarios with our proposed scheme. This savings in spectrum can be used for serving other MBSFN, single cell MBMS or unicast bearers, and it can also be used for increasing the video quality of the same MBSFN bearer.

The paper is organized as follows. Background and related work are discussed in Section 2. The proposed schemes for unicast and multicast are presented in Section 3. Simulation models used to validate the proposed schemes are presented in Section 4. Simulation results are presented and analyzed in Section 5, and conclusions are made in Section 6.

\section{Background and Related Work}

LTE improves the Universal Mobile Telecommunications System (UMTS) mobile phone standard and provides an enhanced user experience for the next generation mobile broadband. Orthogonal Frequency Division Multiple Access (OFDMA) is selected as the physical layer access technology for the LTE downlink. The OFDMA design allows high performance in frequency selective fading channels. Figure 1 illustrates the overall architecture of real-time video delivery over LTE cellular networks, in which the network is comprised of the access network and the core network, known as evolved UMTS terrestrial radio access network (EUTRAN) and evolved packet core (EPC) respectively. Some of the challenges associated with video delivery over 4G networks are presented in [9]. There are many driving factors for improved video support in LTE networks. The most important one is the high throughput possible with LTE technology, which is made possible by the following system design decisions.

Scalable channel bandwidth: LTE supports bandwidths from 1.4 MHz to $20 \mathrm{MHz}$.

Dynamic modulation: A wide range of modulation schemes from Quadrature Phase Shift Keying (QPSK) to 64 Quadrature Amplitude Modulation (QAM) are possible in LTE downlink.

Multiple antenna technology: LTE supports up to 4X4 Multiple Input Multiple Output (MIMO) antenna configurations.

OFDMA: OFDMA in LTE downlink allows radio resources to be allocated in time and frequency domain. This gives link and channel aware schedulers more flexibility for the efficient use of radio resources. 


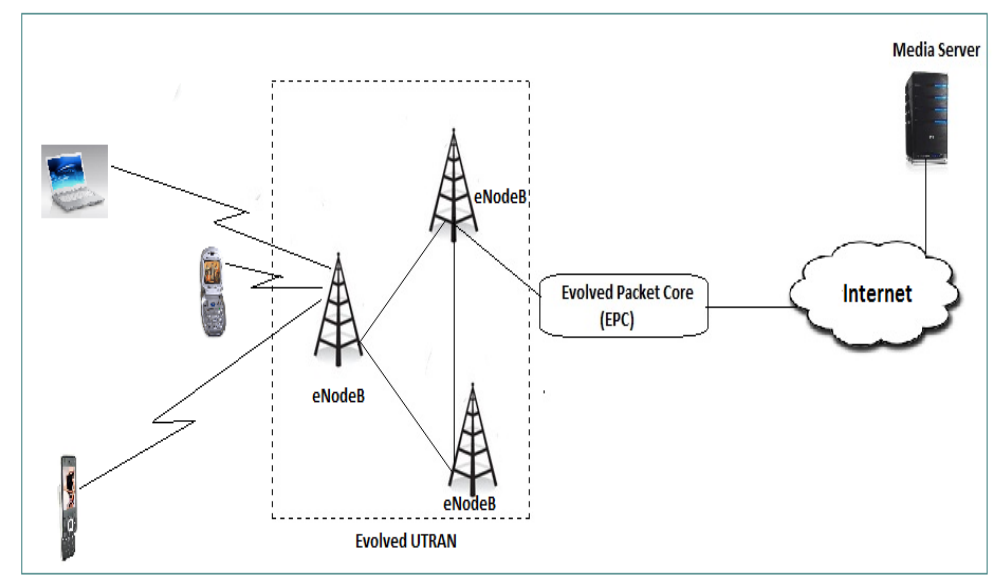

Figure 1: Real time video delivery over LTE

Video transmission with the efficient use of expensive radio resources is very important to mobile operators to support more mobile users with acceptable QoE. Video encoders and cross layer design in LTE access networks play a crucial role in delivering video with the least possible bandwidth and with acceptable quality. H.264 video coding increases coding efficiency by approximately 50\% as compared to previous MPEG (Motion Picture Experts Group) standards. This is achievable using advanced coding techniques, such as multiple reference frames and generalized B-pictures that exploit redundancies over longer time horizons as well as Intra picture prediction of macro blocks that exploits spatial redundancies in a given video frame [10]. 3GPP recommends the use of H.264/AVC baseline profiles for all video based services, like conversational, packet switched streaming services (PSS), Multimedia Messaging Service (MMS) and MBMS services. H.264/AVC baseline profiles are used for delivering video streaming services in Third Generation (3G) networks. But, LTE networks need to support mobile devices with different display resolution requirements like small resolution mobile phones and high resolution laptops. Recognizing this trend, 3GPP research has started focusing on the use cases for advanced video support in LTE networks and on the evaluation of different video codecs. As a result of the 3GPP Release 9 work, a technical report that collects the identified use cases and solutions has been produced [11]. These solutions are based on SVC encoding of videos. SVC has been defined as an extension to the H.264 video coding standard and supports mainly three different types of scalability: spatial scalability, temporal scalability, and quality scalability. Using temporal scalability, the same video sequence can be encoded to support different frame rates. Spatial scalability, on the other hand, is the most important scalability type in SVC. Using spatial scalability, same video can be encoded in multiple resolutions and send using only one bit stream. Spatial scalability is an important tool in wireless networks that support heterogeneous UE types. Finally, quality scalability produces video bit streams with different quality layers, which can be used for the dynamic adaptation of video quality and bit rate based on user and network conditions. SVC allows different types of scalabilities to be encoded in the same bit stream

Adaptive video streaming over wireless networks has been studied from different perspectives in the past. Mobile video transmission over wireless links using SVC is presented in [12]. The integration of SVC into mobile multimedia networks is discussed by the authors. A 
scalable video streaming system using SVC over mobile WIMAX networks is presented in [13]. In this paper, video adaptation is done based on the bandwidth availability of the receiver. Audio and video media coding standards for LTE are discussed in [14]. The objective quality assessment of multidimensional scalability of SVC videos is discussed in [15]. According to the authors, conventional objective quality assessment tools like Peak-Signal-to Noise Ratio (PSNR) fail for scalable video.

Most of these papers discuss the adaptations happening in the MAC layer of the base station. But, dropping of packets in the eNodeB is not a common solution for congestion in backhaul routers and wireless medium in a LTE system. Dropped packets in the eNodeB are waste of resources in LTE backhaul and core network. Dropping video frames in the RTP layer of video server reduces the congestion both in eNodeB and backhaul router. Adaptations in video server using channel quality information of LTE networks are not done to the best of our knowledge. Because of this, we propose in this paper an RTP based bit stream adaptation scheme for SVC encoded traffic. Our adaptation scheme is applicable for unicast video on demand delivery systems with LTE wireless access. Delivering acceptable Quality of Service (QoS) to the maximum number of users in a cell coverage area with minimum usage of radio and other network resources is the goal of this adaptation scheme.

\subsection{Channel Quality in LTE}

In the LTE, subcarriers are assigned to users in the chunks called physical resource blocks. Upon receiving a downlink signal, the UE should report its Signal to Noise Ratio (SNR) to eNodeB via upload control channel. These reports are called CQI reports. CQI values are evaluated from SNR measurements in the UE. CQI value indicates the highest modulation and the code rate at which the block error rate (BLER) of the channel being analyzed does not exceed $10 \%$. Compared to 3GPP UMTS systems, an advanced CQI feedback system is implemented for 3GPP LTE. Sub-band and wideband CQI reports can be obtained from the UE when CQI reporting scheme is enabled. Sub-band CQIs give channel state information for each sub-band, and wideband CQI gives average channel quality information for the entire spectrum. Sub-band CQIs are used for link adaptation purpose and channel dependent scheduling. There are two CQI reporting modes used in LTE.

- aperiodic feedback: UE sends CQI only when it is requested by the Base Station (BS).

- periodic feedback: UE sends CQI periodically to the BS; the period between 2 consecutive CQI reports is communicated by the BS to the UE at the start of the CQI reporting process.

An approximate mapping between SNR and CQI is given in [16] and shown below in Figure 2. 

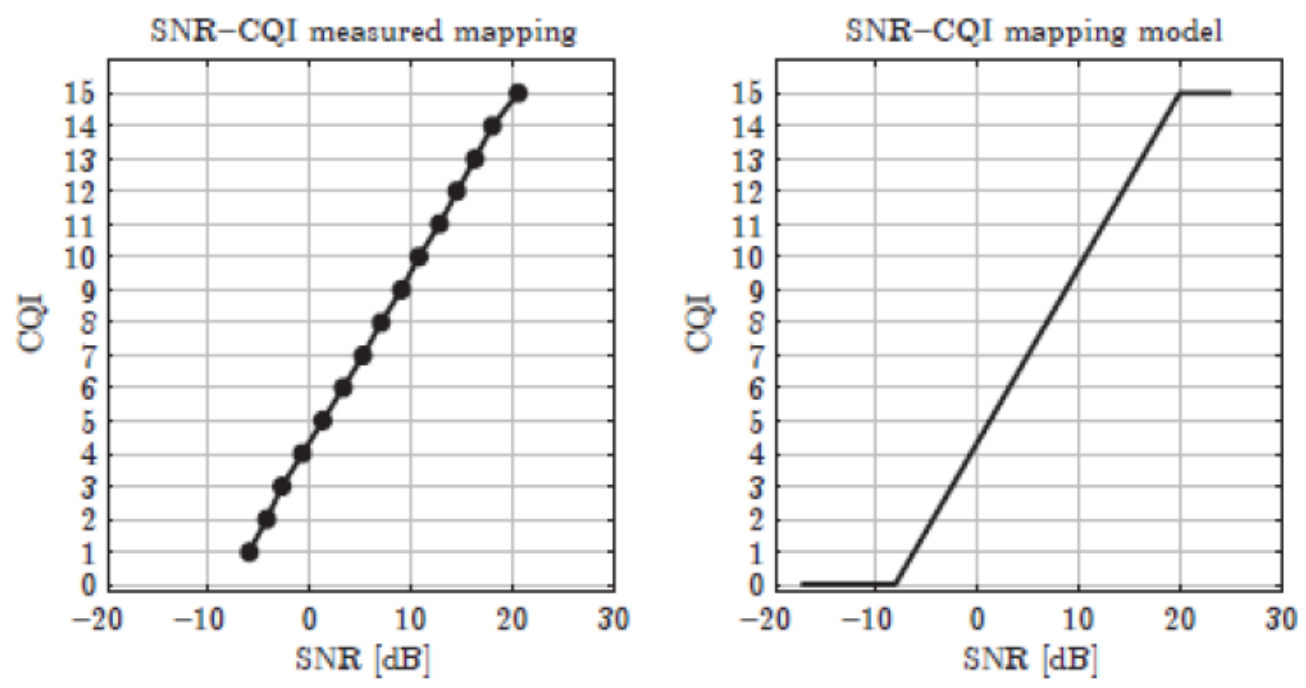

Figure 2: SNR to CQI mapping [16]

\subsection{MBSFN in LTE}

The LTE MBSFN system architecture is shown in Figure 3. Within E-UTRAN, the eNodeBs or base stations (e-NBs) are the collectors of the information that has to be transmitted to users over the air-interface. The Multi-cell/multicast Coordination Entity (MCE) coordinates the transmission of synchronized signals from different cells (e-NBs). MCE is responsible for the allocation of the same radio resources, used by all e-NBs in the MBSFN area for multi-cell MBMS transmissions. Besides allocation of the time/ frequency radio resources, MCE is also responsible for the radio configuration, e.g. the selection of the MCS. The e-MBMS Gateway (eMBMS GW) is physically located between the evolved Broadcast Multicast Service Center (eBMSC) and e-NBs, and its principal functionality is to forward the MBMS packets to each e-NB transmitting the service. The e-MBMS GW is logically split into two domains. The first one is related to control plane while the other one is related to user plane. Likewise, two distinct interfaces have been defined between e-MBMS GW and E-UTRAN, namely M1 for user plane and M3 for control plane. M1 interface makes use of IP multicast protocol for the delivery of packets to e-NBs. M3 interface supports the MBMS session control signaling, e.g. for session initiation and termination. The e-BMSC is the entity in charge of introducing multimedia content into the LTE network. For this purpose, the e-BMSC serves as an entry point for content providers or any other broadcast/multicast source which is external to the network [5].

Scheduling of Multicast Transport Channel $(\mathrm{MCH})$ is done in MCE, whereas unicast scheduling is done in eNodeB. Sub frames used for a MBSFN bearer is changed dynamically in each MCH Scheduling Period (MSP). In MBSFN, the MCE allocates radio resources to the $\mathrm{MCH}$, by the means of an MSAP (MBMS Sub frame Allocation Pattern). Channel estimation of MBSFN users is done using MBSFN reference signals instead of cell-specific reference signals in unicast transmission. Since aggregated channel of all cells involved in the MBSFN transmission will be highly frequency selective, MBSFN reference signals are repeated more frequently compared to cell-specific reference signals. 


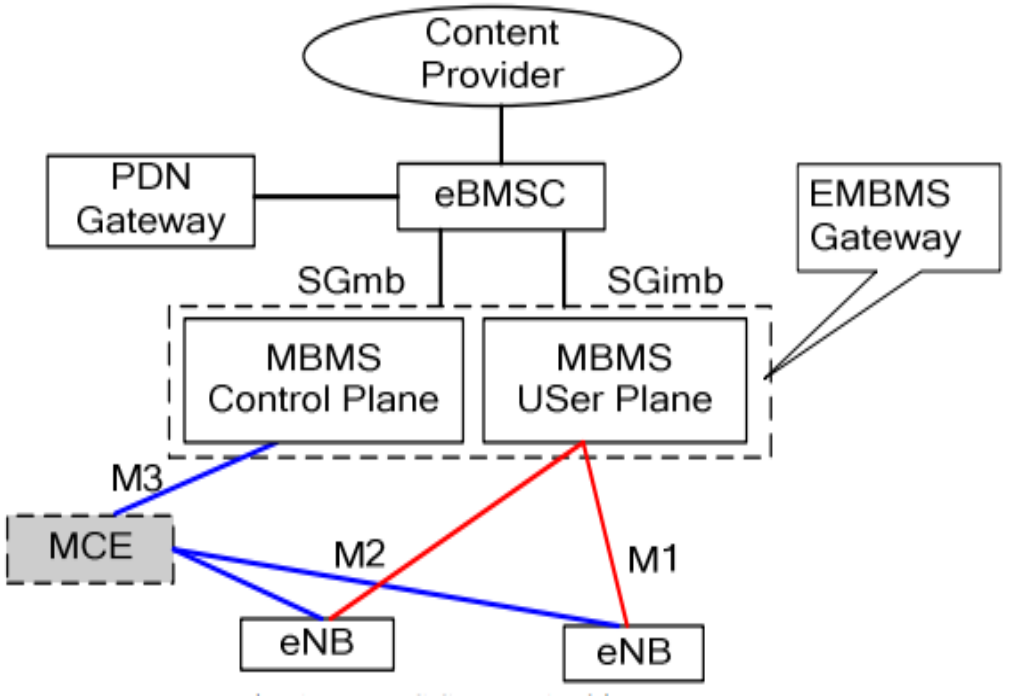

Figure 3: LTE MBSFN system architecture [17]

Authors of [18] present a packet scheduler for UMTS MBMS services. This packet scheduler is designed to deliver two different quality of service for users located in different locations of the same sector of a cell and power requirement of MBMS service is reduced with this. SE refers to the information rate that can be transmitted over a given bandwidth in a specific communication system. It constitutes a measure of how efficiently a limited frequency spectrum is utilized. This can be increased by using high modulation and coding index. Authors of [19] compare SE of MBSFN based LTE networks using different MCS selection algorithms. A typical unicast transmission will have 14 OFDM symbols per sub frame. The Cyclic Prefix (CP) used for MBSFN is longer than CP used in unicast, and this enables the UE to combine transmissions from different eNodeBs located far away from each other. Because of this, only 12 OFDM symbols per sub frame are used for MBSFN and only one sub frame per radio frame is allowed to use for one MBSFN bearer. Moreover, MBSFN reference signals are transmitted more frequently compared to cell-specific reference signals used for unicast transmission. These factors reduce the SE compared to unicast and total cell MBSFN SE calculations.

\section{Proposed Adaptation and Scheduling Schemes for Unicast and Multicast Video Transmissions}

An efficient scheme for channel quality based rate adaptation for unicast SVC video transmission is presented in Section 3.1. AMC and scheduling scheme for MBSFN video transmission is presented in Section 3.2.

\subsection{Unicast Adaptation and Scheduling Scheme}

Channel quality based adaptive bit rate selection scheme for video transmission over LTE network is proposed in this section. Adaptive bit rate is achieved using quality and temporal scalability features of SVC encoding. CQI reports from User Equipments (UEs) are used for the adaptation purpose, and this scheme is aimed at improving video quality of low and medium 
quality users and reducing the bandwidth consumption of expensive wireless spectrum. Packet losses in an end to end video traffic system with LTE wireless access can be of three types: packet losses in backhaul router due to congestion, packet losses in eNodeB due to congestion in the eNodeB buffer, and packet losses in the wireless medium. Packets sent from eNodeB to wireless medium, which will be dropped in the wireless medium due to bad channel quality or will be dropped at the receiver due to less processing power or battery power, is a waste of radio resources.

As discussed in Section 2, UEs in LTE network update eNodeB about the channel quality using CQI reports. For adapting to fast channel quality variations, periodic CQI reporting scheme is used with a reporting interval of $2 \mathrm{~ms}$. The MAC layer in the eNodeB provides CQI information for each user to the RTP layer in the video server. Since RTP is not aware of resource management in the frequency spectrum, sub-band CQI information is not useful for adaptations in RTP layer. So, wideband CQI values are used for the adaptation purpose in video server. Since adaptations for each and every CQI is not practical and not possible to implement in real time systems, adaptations are performed over a group of CQIs. Our proposed adaptation scheme is shown in Figure 4.

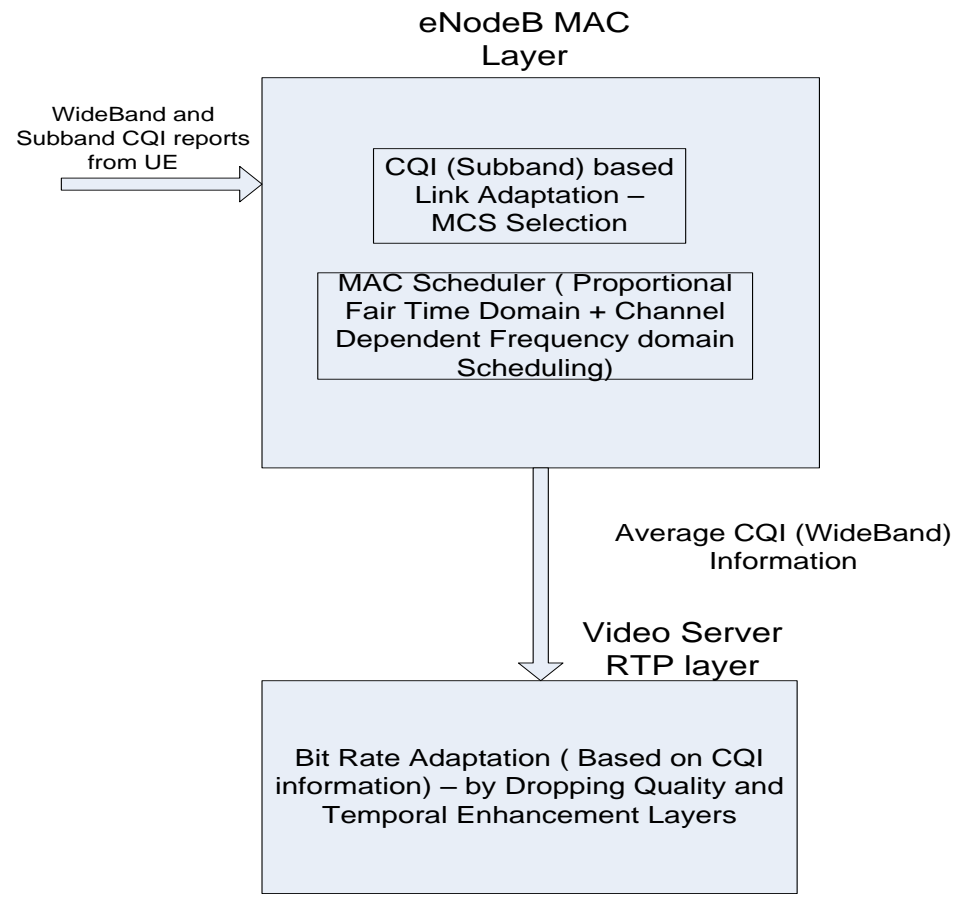

Figure 4: Channel quality based bit rate adaptation in the video server

Based on the CQI, Modulation and Coding Scheme (MCS) scheme for the user is changed dynamically. A two-level scheduler is used in the eNodeB MAC to ensure fairness among users and to exploit channel diversity of users. A proportional fair time domain scheduler is used for video bearers, and channel state dependent frequency domain scheduling is used to exploit multiuser diversity in the fading wireless medium. Sub-band CQI reports are used in the scheduler to allocate preferred sub-band to each user. Proposed scheduling scheme in the MAC layer is 
shown in Figure 5. SVC adaptation scheme used for different channel qualities are as shown in Table 1. Quality and temporal scalability features of SVC bit stream are used for adaptation. Bit rate possible for channel quality of the user is measured statically and used in this dynamic adaptation. Quantization parameter $(\mathrm{QP})$ for base quality layer is selected based on the bit rate possible for low quality user. Similarly, QPs for two enhancement quality layers are selected based on the bit rate possible for medium and high quality users.

Table 1: Proposed SVC adaptation scheme in the video server

\begin{tabular}{l|l}
\hline CQI Range & SVC Adaptation \\
\hline $10-15$ ( High Quality Channel) & Three Quality Layers and three temporal Layers \\
$7-9$ ( Medium Quality Channel) & Two Quality Layers and two temporal layers \\
$1-6$ ( Low Quality Channel) & One Quality Layer and one temporal layer \\
\hline
\end{tabular}

Our scheme is also aimed at avoiding the over provisioning of radio resources in eNodeB. Since SVC video encoding produces highly variable bit rate traffic, assigning a high GBR value is the only solution for avoiding buffer overflow in eNodeB during congestion. However, instead of assigning same GBR values to all users in the cell coverage area, we suggest the use of CQI value of UEs for assigning GBR values. This helps to assign only the required resources to each user, and valuable wireless spectrum resources can be saved. Statically measured achievable bit rate for each CQI range (in specific channel conditions) is used as the GBR value for users.

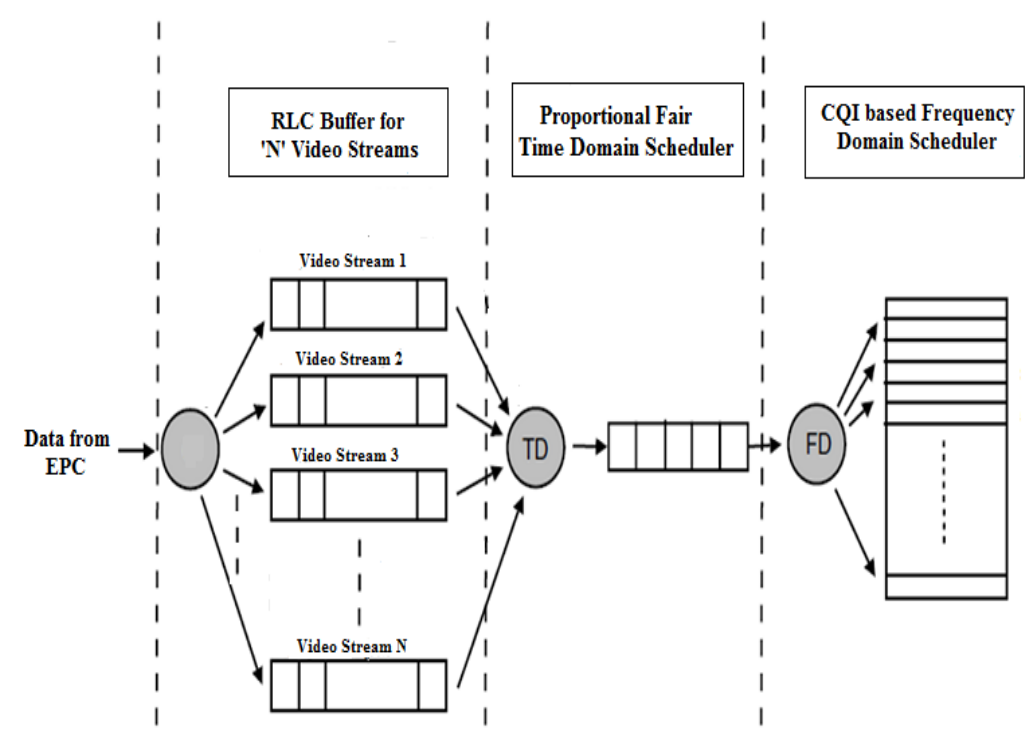

Figure 5: MAC scheduling scheme in the eNodeB

\subsection{Multicast Adaptation and Scheduling Scheme for MBSFN}

An AMC and scheduling scheme is proposed for providing graceful degradation of video quality to users in MBSFN area and spectrum savings of MBSFN bearer. SINR values measured 
at UEs are used for adaptation of MCS for base layer (BL) and enhancement layers (EL). MCS and scheduling of base layer and enhancement layers are changed based on distribution of users in high, medium and low channel quality regions. Since SINR measurements are done periodically, changes in user distribution (e.g. mobile users and new users) are considered in this scheme. This adaptation scheme increases the spectrum efficiency of MBSFN channel and allows serving users with maximum throughput possible in each channel quality region. Three layers of SVC video are split into 3 sub-channels in the physical layer. This gives tight control for physical layer adaptation and scheduling of each video layer separately. UEs are informed about the frequency range of each layer to allow efficient and fast decoding. This scheme ensures coverage of all the users inside MBSFN area and provides graceful degradation of video quality based on channel quality.

MCS selection and scheduling algorithm for BL, EL1and EL2 of the SVC video in MCE is given below as Algorithm 1 and decoding procedure in UEs is given below as Algorithm 2. Channel allocation of multicast channel for different layers is shown in Figure 6. F1- F2, F2-F3 and F3-F4 are the frequency ranges allocated for BL, EL1 and EL2, respectively, of SVC video by MCE.

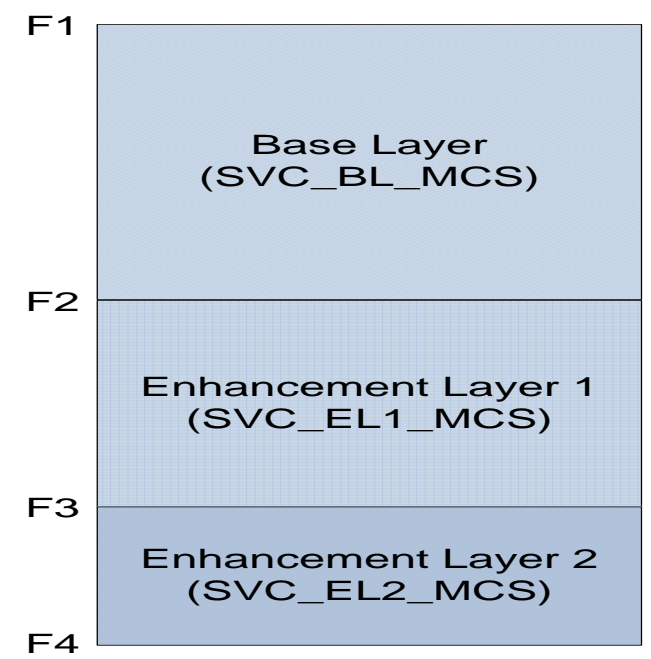

Figure 6: Layered transmission of SVC video in MBSFN

\footnotetext{
Algorithm 1. MBSFN dynamic scheduling and AMC algorithm in MCE

1: Measure SINR for users distributed in MBSFN area

2: Calculate MCS value for each SINR value for providing coverage in that specific SINR region

3: Group SINR values to three channel quality regions and MCS values for low, medium and high channel quality regions are measured. MCS value for each group is selected as the low MCS value in that group to ensure coverage for all users in that group.

4: for each UE $v$ in MBSFN Area calculate $\operatorname{SINR}(\mathrm{v})$

5: if $\operatorname{SINR}(\mathrm{v})$ is member of low channel quality

6: $\quad$ Increment the count of low channel quality users

7: else if $\operatorname{SINR}(v)$ is member of medium channel quality
} 
8: $\quad$ Increment the count of medium channel quality users

9: else if $\operatorname{SINR}(v)$ is a member of high channel quality

10: Increment count of high channel quality users

11: end if

12: end for

13: Set MCS of BL video (SVC_BL_MCS) to high

14: Set MCS of EL1video (SVC_EL1_MCS) to high

15: Set MCS of EL2 video (SVC_EL2_MCS) to high

16: if Medium channel quality count $>0$

17: Set SVC_EL1_MCS to medium

18: Set SVC_BL_MCS to medium

19: end if

20: if Low channel quality count $>0$

21: Set SVC_BL_MCS to low

22: end if

23: if High channel quality count $>0$

24: $\quad$ Schedule BL, EL1 and EL2 for transmission

25: else if Medium channel quality count $>0$

26: $\quad$ Schedule BL and EL1 for transmission

27: else if Low channel quality count $>0$

28: $\quad$ Schedule BL for transmission

29: end if

30: Assign frequency ranges F1, F2, F3, F4 to corresponding SVC layers as shown in Figure 6

31: Send F1, F2, F3, F4 and MCS scheme to all UEs in the MBSFN area

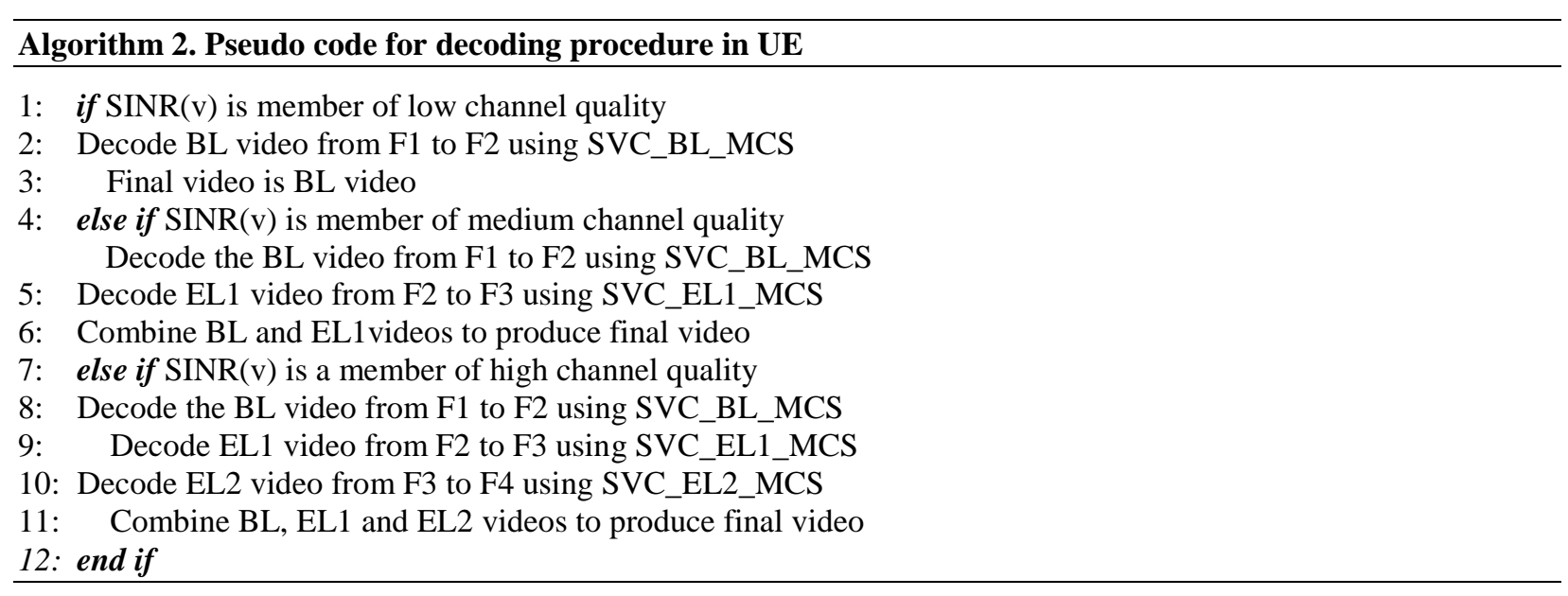

This scheme is expected to reduce the spectrum usage in scenarios with no users in low and medium channel quality regions. This dynamic spectrum saving can be used for accommodating other MBSFN bearers or unicast bearers with Best Effort (BE) service model. Another option is to increase the bit rate for medium and high quality users in the same MBSFN bearer to obtain higher video quality. This scheme can be implemented with minimal change in MCE and eNodeB nodes of LTE networks. Further, this scheme can be adapted to multicast and broadcast services in other OFDMA based wireless networks such as WiMAX Multicast Broadcast Service (MCBCS) single frequency operation [20]. 
4. Simulation Methodology

OPNET simulation models for analysis of proposed schemes are discussed in this section.

\subsection{Unicast Simulation Model}

Single cell network is used for the analysis of the proposed scheme, considering no inter-cell interference or handover scenarios. Infinite bandwidth model is used in the core network links and routers. Network topology used for simulations is shown in Figure 7. Simulations are done using Star Wars 4 movie video trace from [21]. Since LTE needs to support heterogeneous receivers including high resolutions with high quality video, high quality SVC video stream is used for the analysis purpose. SVC encoder setting used is shown in Table 2. Rate distortion curve is non-linear for all videos available in [21] and it is a general property for a SVC bit stream. Rate Distortion curve for Star Wars SVC bit stream is shown below in Figure 8. LTE system configuration used in the simulation is given below in Table 3.

Table 2: SVC encoder settings

\begin{tabular}{l|l}
\hline Parameters & Value \\
\hline Number of Frames & 500 \\
Resolution & CIF ( 352 X 288) \\
Frame Rate & $30 \mathrm{fps}$ \\
GOP & 16 \\
Number of B Frames & 3 \\
between I and P Frames & \\
Encoding Bit Rate & VBR \\
GOP Structure & IBBBPBBBPBBBPBBB \\
SVC scalability layers & 3 Quality and 3 Temporal Layers \\
\hline
\end{tabular}

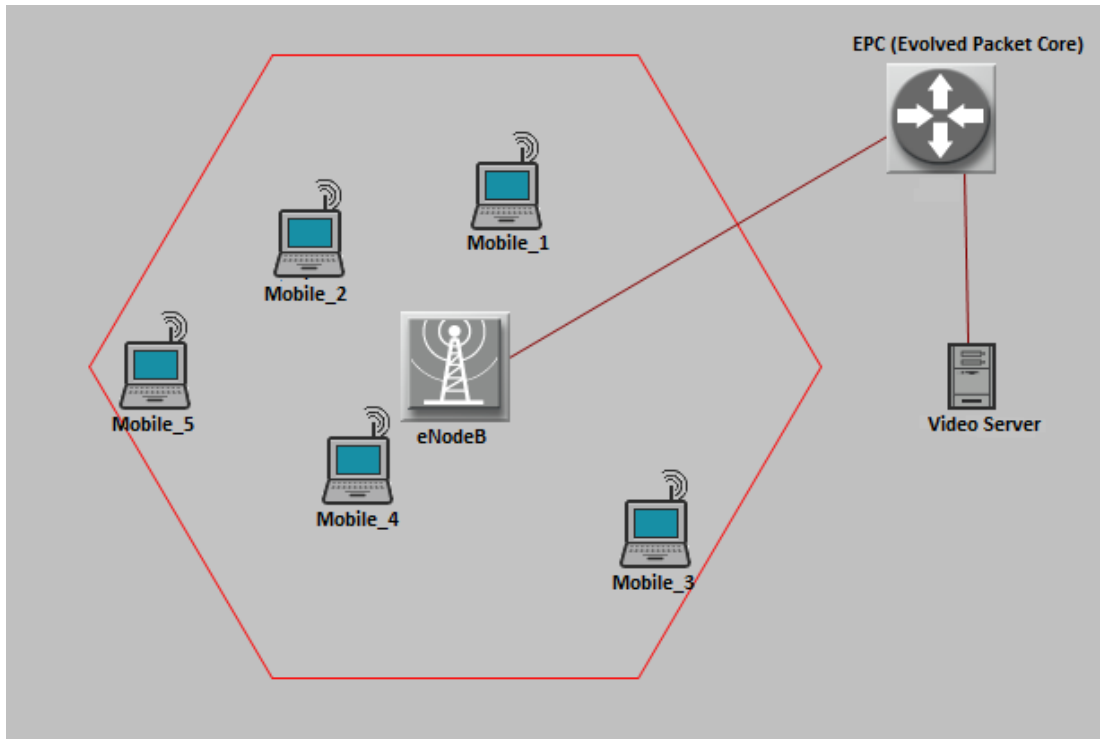

Figure 7: OPNET [8] LTE simulation model 


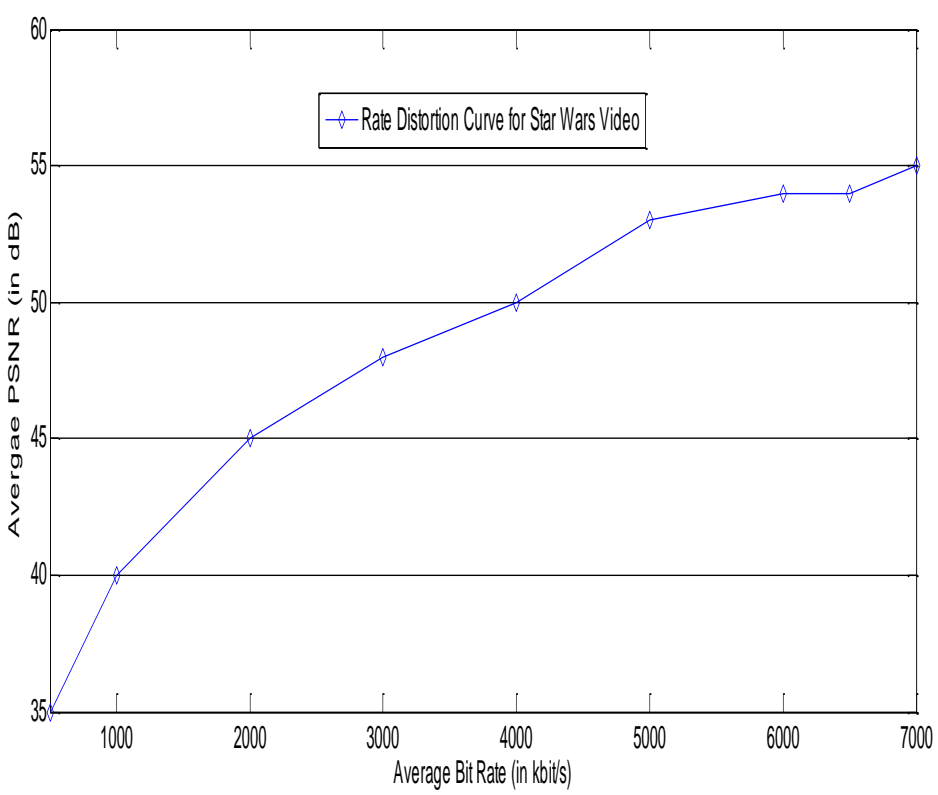

Figure 8: Rate Distortion Curve for Star Wars Movie video

PSNR is one of the most popular objective quality measures used for video quality. But, PSNR cannot show properly the effect of prediction errors (lost I- and P-pictures) that may be highly visible in the video distortion, but it will create little distortion in terms of mean square error. As explained in [22], PSNR cannot be used for quality prediction in cases of lost video frames over networks. However, it can be used as a quality indicator of the encoded input video for simulation.

Table 3: LTE system parameters

\begin{tabular}{l|l}
\hline Parameters & Value \\
\hline LTE Mode & FDD \\
eNodeB Operating Power & $46 \mathrm{dBm}$ \\
Carrier Frequency & $2.5 \mathrm{GHz}$ \\
LTE Bandwidth & $10 \mathrm{MHz}$ (50 PRBs) \\
User Location & $\begin{array}{l}\text { Users with different Channel Qualities (High, Medium } \\
\text { and Low) equally distributed in the cell coverage area }\end{array}$ \\
MAC Scheduler & $\begin{array}{l}\text { Proposed Scheduler (PF in TD and Channel Dependent } \\
\text { in FD) }\end{array}$ \\
Path Loss Model & Suburban MacroCell \\
Multipath Channel Model & ITU Pedestrian A \\
\# of Tx Antennas & 1 \\
\# of Rx Antennas & 1 \\
CQI reporting Scheme & Wideband (Used for RTP adaptation) + Subbands (Used \\
& for MAC Scheduling) - Periodic reporting Scheme (2 \\
RLC Mode & UM interval) \\
Maximum number of & 3 \\
HARQ retransmissions & \\
\hline
\end{tabular}


To evaluate the quality of an output video sequence, we adopt an evaluation model of video quality (Q) from [23], which is defined by the number of decodable frames over the total number of frames originally sent from the video source.

$$
Q=\frac{N_{\text {dec }}}{N_{\text {total }-I}+N_{\text {total }-P}+N_{\text {total }-B}},
$$

where $0<\mathrm{Q}<1, N_{d e c}$ is the total number of decodable frames including all three types of frames (i.e., $\left.N_{d e c}=N_{d e c-I}+N_{d e c-P}+N_{d e c-B}\right)$. It is assumed that dependencies between different types of frames (i.e., I, P, and B frames) are already considered in the derivation of the numbers of different decodable frames. $N_{\text {total-I }}, N_{\text {total-P }}$ and $N_{\text {total }-B}$ are the total number of I-, P-, B-frames in the video source, respectively. I (Intra-coded) frames are encoded independently and decoded by themselves. P (Predictive-coded) frames are encoded using predictions from the preceding I or P frame in the video sequence. B (Bi-directionally predictive-coded) frames are encoded using predictions from the preceding and succeeding I or P frames. The loss of an I frame is essentially equivalent to the loss of all the frames in the Group of Pictures (GOP). B frames are not used for decoding any other frames. The above mentioned dependencies are used in the calculation of $N_{\text {dec-I}}, N_{\text {dec-P }}$, and $N_{\text {dec_B }}$. Dependencies of different type of frames inside one GOP is shown below in Figure 9.

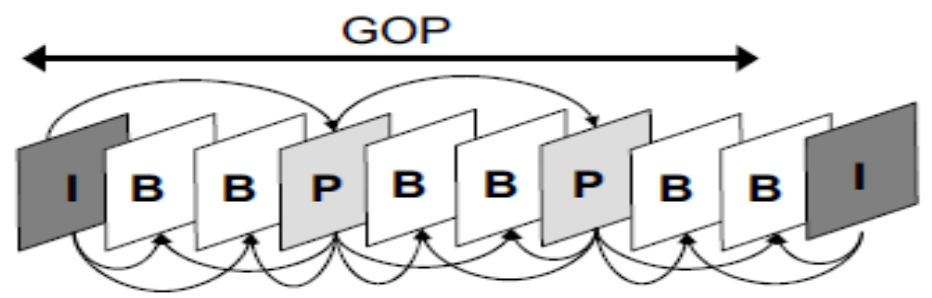

Figure 9: Dependencies of Frames in a SVC GOP Structure with size 10.

$\mathrm{Q}$ is an objective measure to evaluate the video quality. The larger $\mathrm{Q}$ means the better video quality is perceived by the recipient. Since our adaptation method uses different bit streams for throughput and quality adaptations, this objective quality measure needs to be multiplied with input PSNR value to get a reliable objective quality measure for output bit stream.

In other words,

$$
\text { Output video quality measure }=\mathrm{Q}^{*} P S N R_{\text {input_video }} \text {, }
$$

The following scenario is simulated to prove the benefits of CQI based adaptation in the video server. Suburban macrocell scenario with $3 \mathrm{~km}$ cell radius is used for simulations. Three users from different channel quality regions with CQI Low (3-4), CQI Medium (7-8) and CQI High (12-13) are used in the analysis. Distributions of these three users are as given below in Table 4. 
Table 4: Mobile user distribution

\begin{tabular}{c|c|l|l}
\hline User & Distance from eNodeB $(\mathbf{K m})$ & MCS Wideband Index & CQI \\
\hline Mobile_1 & $0.2-0.5$ & $23-24$ & $12-13$ \\
Mobile_2 & $1-1.5$ & $13-14$ & $7-8$ \\
Mobile_3 & $2.5-3$ & $5-7$ & $3-4$ \\
\hline
\end{tabular}

\subsection{Multicast (MBSFN) Simulation Model}

MBSFN area is created with 7 cells organized as shown in Figure 10. UE measurements are done on entire cell coverage area of eNodeB_1, and other surrounding cells assist eNodeB_1 in MBSFN operation. SVC video traces are created using JSVM SVC encoding software [24]. SVC encoder setting used is shown in Table 5. System configuration used in the simulation is given in Table 6. Users in the MBSFN area is divided into 3 channel quality regions: High, Medium and Low. Simulations are done with SVC trace of Football video [25] generated using JSVM. Spatially scalable video with QCIF base layer, and CIF enhancement layer is used for the simulations. Since multicast group can contain heterogeneous receivers, this is a valid requirement in the real-time scenario. One base quality layer with data rate of $256 \mathrm{kbps}$, first enhancement layer with $150 \mathrm{kbps}$ and second enhancement layer with $100 \mathrm{kbps}$ are encoded for multicast transmission.

Table 5: SVC encoder settings

\begin{tabular}{l|l}
\hline Parameters & Value \\
\hline Number of Frames & 500 \\
Resolution & QCIF Base Layer and CIF Enhancement layer \\
Frame Rate & $30 \mathrm{fps}$ \\
GOP & 16 \\
SVC scalability layers & 2 Spatial layers and 3 Quality layers. \\
\hline
\end{tabular}

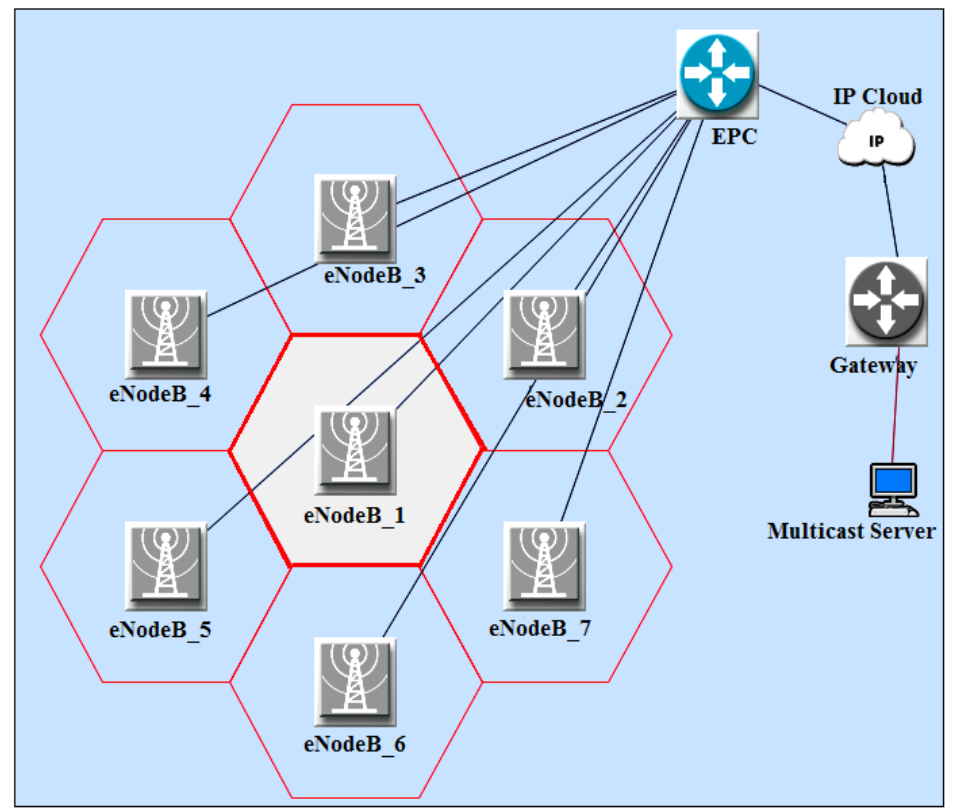

Figure 10: MBSFN Network diagram used for simulation 
Table 6: LTE system parameters

\begin{tabular}{l|l}
\hline Parameters & Value \\
\hline LTE Mode & FDD \\
eNodeB Operating Power & $46 \mathrm{dBm}$ \\
Inter Site Distance & 1732 meter \\
$\begin{array}{l}\text { Number of OFDM symbols } \\
\text { per subframe (1 ms) }\end{array}$ & 12 \\
Cyclic prefix & $16.6 \mathrm{us}$ \\
Carrier Frequency & $2.5 \mathrm{GHz}$ \\
LTE Bandwidth & $20 \mathrm{MHz}$ (100 PRBs) \\
User Location & $\begin{array}{l}\text { Users with different Channel Qualities } \\
\text { (High, Medium and Low) equally }\end{array}$ \\
Path Loss Model & distributed in the MBSFN area \\
Multipath Channel Model & Suburban MacroCell \\
\# of Tx Antennas & ITU Pedestrian A \\
\# of Rx Antennas & 1 \\
\hline
\end{tabular}

\section{Simulation Results and Analysis}

\subsection{Unicast Adaptation Scheme}

Simulation results for the proposed scheme for unicast video transmission are presented and analyzed in this section. As expected, CQI values of users fluctuated over simulation time. This is mainly due to the multipath fading enabled LTE network model. MCS Index over simulation time for medium quality user (Mobile_2) is given below in Figure 11 as an example. This confirms our hypothesis as to why CQI adaptation is not done for each CQI feedback in the video server and why wideband CQI is used for the adaptation purpose. Because of the high CQI fluctuation, feedback to video server and adaptation in real time is not possible. We take long time average CQI values for the adaptation purpose.

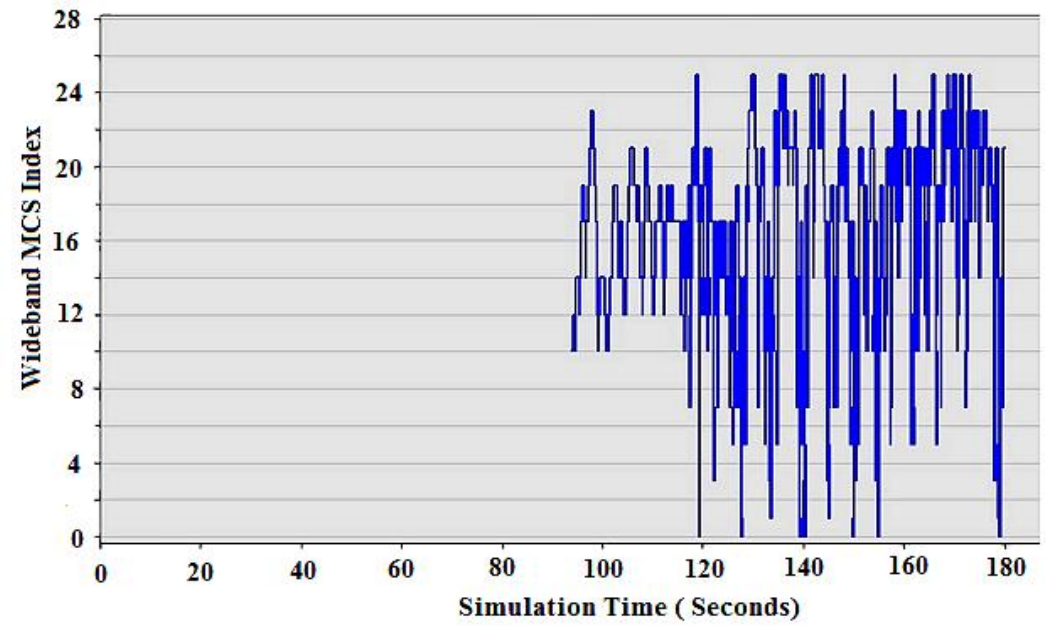

Figure 11: Channel quality (MCS Index) fluctuation for medium channel quality user 
Objective video quality measured using Eqn. (1) for three CQI values without adaptation is given in Figure 12. CQI based adaptation is used for medium and low quality channel users and the resulting video qualities are compared with the results obtained without adaptations in Figure 13 and 14. Objective video quality gains are very much visible from this comparison. From results summarized in Table 7 and 8, we can see that PSNR of input bit stream is almost the same for high and medium quality video. But, average output video quality is improved with considerable reduction in bit rate. PSNR of high quality, medium quality and low quality sent to three different users are shown below in Figure 15. PSNR of low quality user is selected above $35 \mathrm{~dB}$ to ensure good input video quality based on PSNR to MOS mapping. We can see that approximately $13 \%$ video quality gain can be obtained for low channel quality user with adaptation. Packet loss ratios for low, medium and high channel quality users with and without adaptation are compared in Figure 16 and 17 respectively.

As we can see from the results, packet loss ratio is decreased and video quality is increased with the removal of quality and temporal layers based on the CQI value. Of course, this comes at the cost of reduced quality video from the video server. But, video quality is not linearly reduced with bit rate reduction. This is especially true for high quality input videos. Savings in average bit rate for low and medium quality videos is also noticeable. Without adaptation, remaining throughput (1.5 Mbps for medium quality user and 2.5 Mbps for low quality user) is a waste of radio spectrum.

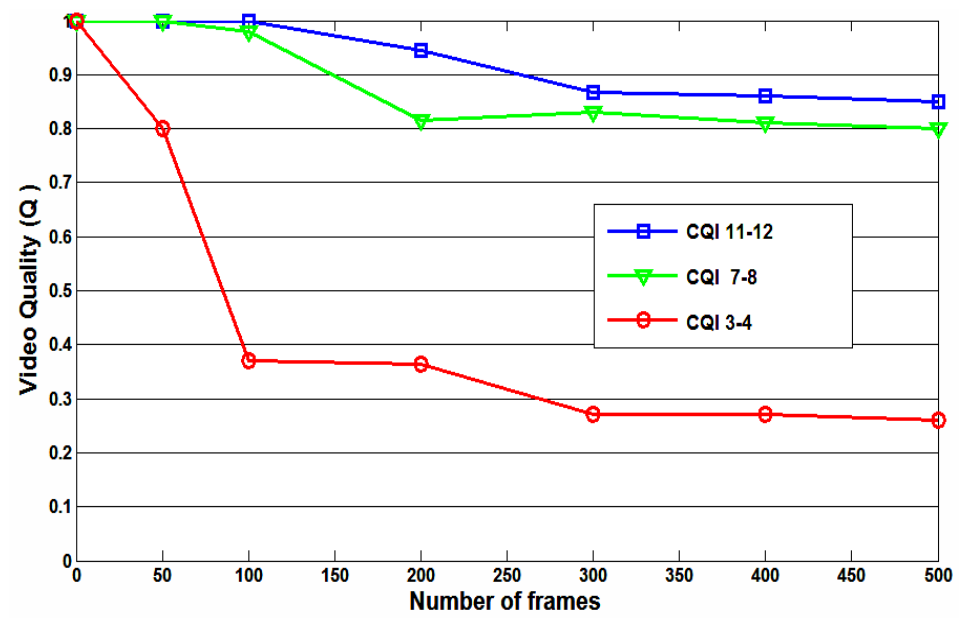

Figure 12: Video quality for different CQI values without adaptation 


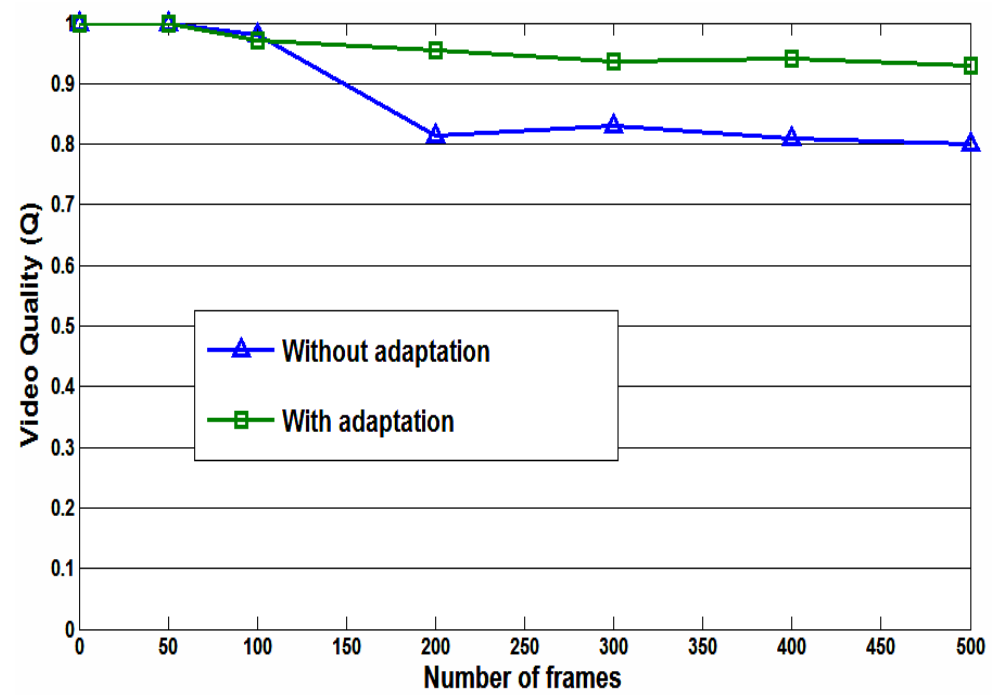

Figure 13. Video quality of medium CQI user (7-8) with and without adaptation

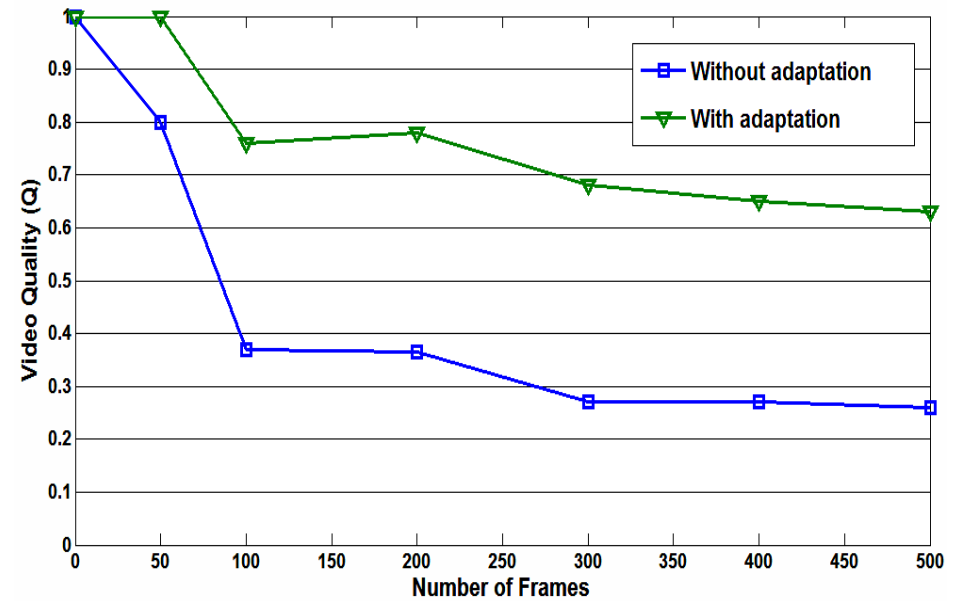

Figure 14: Video quality of low CQI user (CQI 3-4) with and without adaptation

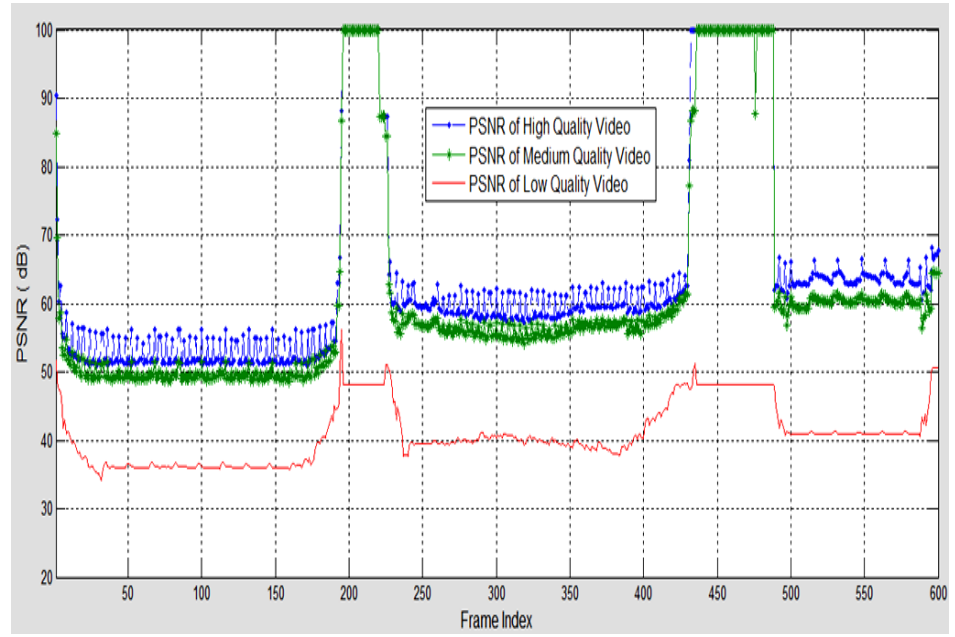

Figure 15: PSNR for High, Medium and Low Quality Videos 


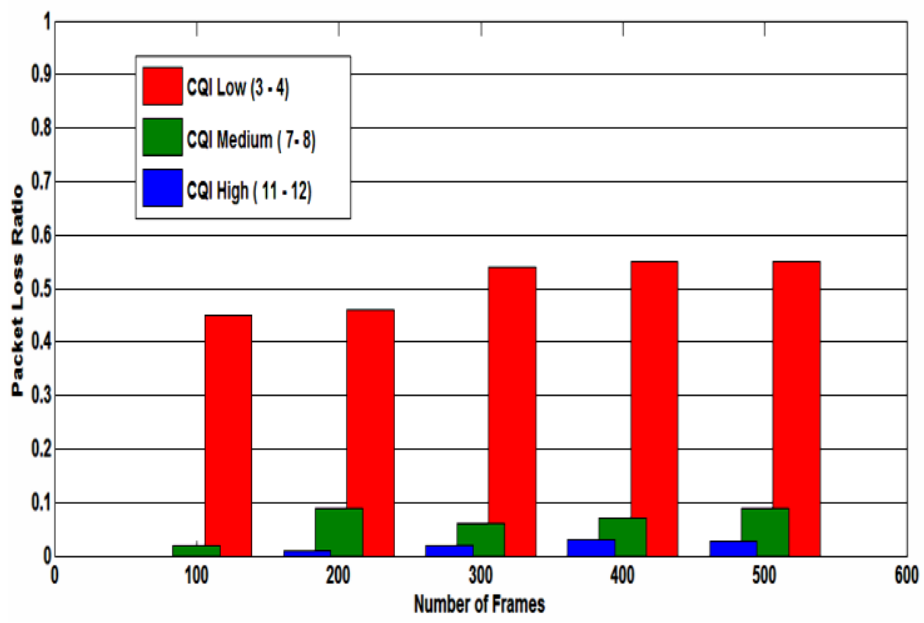

Figure 16: Packet loss ratio for different CQIs wthout adaptation

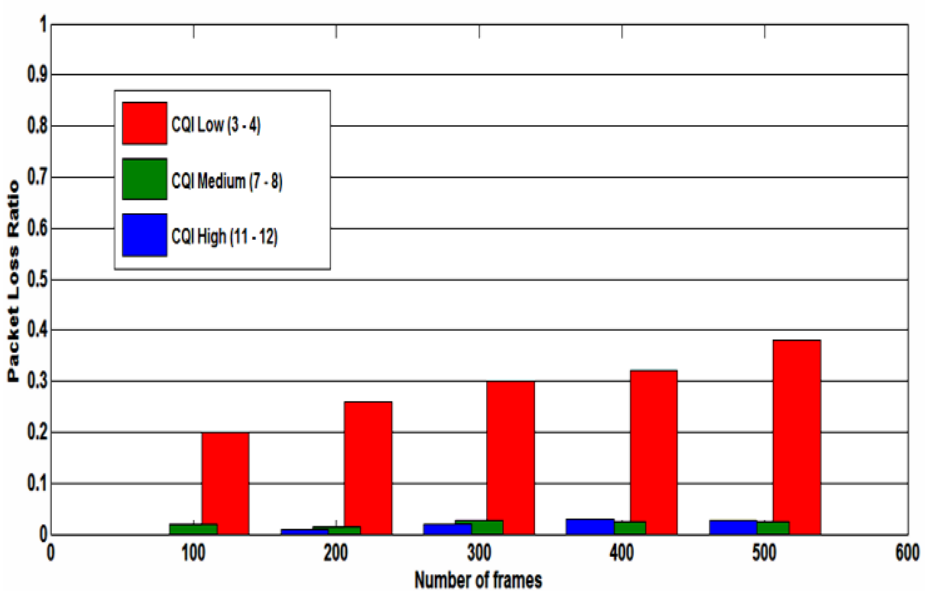

Figure 17: Packet Loss Ratio for different CQIs with adaptation

Table 7: Summary of results without adaptation

\begin{tabular}{|l|l|l|l|l|l|}
\hline $\begin{array}{l}\text { Channel } \\
\text { quality }\end{array}$ & $\begin{array}{l}\text { Avg. objective } \\
\text { video quality (Q) }\end{array}$ & $\begin{array}{l}\text { Avg. packet loss } \\
\text { ratio }\end{array}$ & $\begin{array}{l}\text { Avg. } \\
\text { bit rate }\end{array}$ & $\begin{array}{l}\text { Avg. PSNR of } \\
\text { input video }\end{array}$ & $\begin{array}{l}\text { PSNR*Q (output } \\
\text { video quality) }\end{array}$ \\
\hline High & 0.9 & 0.02 & $3 \mathrm{Mbps}$ & $60 \mathrm{~dB}$ & $54 \mathrm{~dB}$ \\
\hline Medium & 0.85 & 0.08 & $3 \mathrm{Mbps}$ & $60 \mathrm{~dB}$ & $51 \mathrm{~dB}$ \\
\hline Low & 0.4 & 0.45 & $3 \mathrm{Mbps}$ & $60 \mathrm{~dB}$ & $24 \mathrm{~dB}$ \\
\hline
\end{tabular}

Table 8: Summary of results with adaptation

\begin{tabular}{|l|l|l|l|l|l|}
\hline $\begin{array}{l}\text { Channel } \\
\text { quality }\end{array}$ & $\begin{array}{l}\text { Avg. objective } \\
\text { video quality (Q) }\end{array}$ & $\begin{array}{l}\text { Avg. packet } \\
\text { loss ratio }\end{array}$ & Avg. bit rate & $\begin{array}{l}\text { Avg. PSNR of } \\
\text { input video }\end{array}$ & $\begin{array}{l}\text { PSNR*Q (output } \\
\text { video quality) }\end{array}$ \\
\hline High & 0.9 & 0.02 & $3 \mathrm{Mbps}$ & $60 \mathrm{~dB}$ & $54 \mathrm{~dB}$ \\
\hline Medium & 0.93 & 0.02 & $1.5 \mathrm{Mbps}$ & $55 \mathrm{~dB}$ & $51.15 \mathrm{~dB}$ \\
\hline Low & 0.68 & 0.25 & $0.5 \mathrm{Mbps}$ & $40 \mathrm{~dB}$ & $27.2 \mathrm{~dB}$ \\
\hline
\end{tabular}

Our simulations also show that the bit rate possible for each channel quality of the user is highly dependent on path loss model, multipath model and shadow fading margin used in the 
simulation. With $10 \mathrm{MHz}$ LTE system bandwidth, approximately $50 \mathrm{Mbps}$ data can be sent in the downlink. Considering control plane signals and packet header overheads as $20 \%$, only remaining bandwidth can be allocated for actual video traffic. This corresponds to $40 \mathrm{Mbps}$. Without proposed GBR selection scheme, same GBR value is used for all the users independent of channel quality. If we use $3 \mathrm{Mbps}$ GBR for all the users, only 13 users can be accommodated in the cell assuming all the users are accessing same video and no other services are allocated in network. But with CQI based GBR allocation, $3 \mathrm{Mbps}$ is allocated for high quality users, 1.5 Mbps for medium quality users and $0.5 \mathrm{Mbps}$ for low quality users. Considering the equal distribution of users in three channel quality regions, 24 users can be allocated. This helps to admit more number of users and to improve cell coverage.

\subsection{Multicast Adaptation Scheme for MBSFN}

SINR values of users distributed in MBSFN area and MCS value required for providing coverage in that SINR region are measured using simulations. SE achievable with each of this MCS values are also calculated from simulation results. SINR values and corresponding MCS and SE values measured using MBSFN simulations are shown below in Table 9. These SE values are calculated for one MBSFN bearer using specified MCS values. SINR values are grouped to low, medium and high channel quality regions, and low MCS in each group is selected as MCS value for that specific group. These groups, SINR range, and MCS value selected are shown in Table 10 .

Frequency spectrum and number of Physical Resource Blocks (PRB) used by each video layer is calculated and total frequency spectrum utilization is compared for three different channel quality distribution scenarios. One PRB corresponds to $180 \mathrm{Khz}$ of frequency spectrum, and it is the smallest possible allocation block in the frequency domain [5]. MCS is changed for base and enhancement layers based on the channel quality distribution and total spectrum usage is calculated in each scenario. Spectrum savings in Scenario B and Scenario C are calculated and compared with Scenario A (existing approach).

Table 9: SINR, MCS and SE mapping in MBSFN simulation

\begin{tabular}{|l|l|l|l|}
\hline SINR $(\mathbf{d B})$ & MCS Index & Modulation Scheme & SE of MBSFN bearer (bits/second/Hz) \\
\hline-3.85 & 1 & QPSK & 0.02009 \\
\hline-2.1 & 3 & QPSK & 0.03231 \\
\hline-0.35 & 5 & QPSK & 0.05157 \\
\hline 1.4 & 7 & QPSK & 0.07517 \\
\hline 3.15 & 9 & QPSK & 0.10078 \\
\hline 4.9 & 12 & 16QAM & 0.12657 \\
\hline 6.65 & 14 & 16QAM & 0.16407 \\
\hline 8.4 & 16 & 16QAM & 0.20625 \\
\hline 10.15 & 19 & 64QAM & 0.23404 \\
\hline 11.9 & 21 & 64QAM & 0.28477 \\
\hline 13.65 & 23 & 64QAM & 0.33448 \\
\hline 15.4 & 25 & 64QAM & 0.38772 \\
\hline 17.15 & 27 & 64QAM & 0.43845 \\
\hline 18.9 & 28 & 64QAM & 0.47612 \\
\hline
\end{tabular}




\subsubsection{Scenario A: Users are distributed uniformly in cell coverage area (Existing Approach)}

Users are distributed in all channel quality regions in this scenario. This corresponds to previous work on single cell multicast cellular systems with static adaptive modulation and coding selection for video layers [6, 7]. In this case base layer is sent with MCS 1, EL 1 is sent with MCS 12, and EL 2 is sent with MCS 19. These MCS values are selected based on measurements shown in Tables 9 and 10. Table 11 provides the spectrum utilization in number of PRBs and $\mathrm{Hz}$ for different SVC video layers. Since base layer is coded with low MCS value, major number of PRBs (71 PRBs) are used for base layer video, and total spectrum usage is 14.58 MHz in each MBSFN radio subframe.

Table 10: Channel quality regions, MCS and spectral efficiency

\begin{tabular}{|l|l|l|l|}
\hline Channel quality regions & SINR value(dB) & MCS & Spectral efficiency of MBMS bearer (bps/Hz) \\
\hline High & $10.15-18.9$ & 19 & 0.23404 \\
\hline Medium & $4.9-8.4$ & 12 & 0.12657 \\
\hline Low & $-3.85-3.15$ & 1 & 0.02009 \\
\hline
\end{tabular}

Table 11: Spectrum usage with users distributed in all channel quality regions

\begin{tabular}{|l|l|l|l|l|}
\hline SVC video layer & Data rate (kbps) & MCS used & Number of PRBs used & Spectrum Used (MHz) \\
\hline Base & 256 & 1 & 71 & 12.78 \\
\hline Enhancement 1 & 150 & 12 & 7 & 1.26 \\
\hline Enhancement 2 & 100 & 19 & 3 & 0.54 \\
\hline
\end{tabular}

\subsubsection{Scenario B: Users only in high and medium channel quality regions}

Users are distributed across high and medium channel quality regions in this scenario. In this case base layer is sent with MCS 12, EL 1 is sent with MCS 12, and EL 2 is sent with MCS 19. Spectrum utilization is given below in Table 12. Compared to scenario A, there is a drastic reduction in the number of PRBs used for base layer (Only 12 PRBs) as the MCS value is increased from 1 to 12 . Total spectrum usage is $3.96 \mathrm{MHz}$ in each MBSFN radio subframe.

Table 12: Spectrum usage with users distributed only in medium and high channel quality regions

\begin{tabular}{|l|l|l|l|l|}
\hline SVC video layer & Data rate (kbps) & MCS used & Number of PRBs used & Spectrum Used (MHz) \\
\hline Base & 256 & 12 & 12 & 2.16 \\
\hline Enhancement 1 & 150 & 12 & 7 & 1.26 \\
\hline Enhancement 2 & 100 & 19 & 3 & 0.54 \\
\hline
\end{tabular}

\subsubsection{Scenario C: Users only in high channel quality region}

Users are distributed only in high channel quality regions in this scenario. In this case, all video layers are sent with MCS 19. Spectrum utilization in number of PRBs is given below in Table 13. Total spectrum usage is $2.52 \mathrm{MHz}$ in each MBSFN radio subframe. Since high MCS is used for all the layers in this scenario, spectrum savings compared to Scenario A is very high in this case. Savings in radio spectrum for all three scenarios are compared in Table 14. 
Table 13: Spectrum usage with users distributed only in high channel quality region

\begin{tabular}{|l|l|l|l|l|}
\hline SVC video layer & Data rate (kbps) & MCS used & Number of PRBs used & Spectrum Used (MHz) \\
\hline Base & 256 & 19 & 7 & 1.26 \\
\hline Enhancement 1 & 150 & 19 & 4 & 0.72 \\
\hline Enhancement 2 & 100 & 19 & 3 & 0.54 \\
\hline
\end{tabular}

Simulation results for the proposed scheme in Scenarios B and C indicate advantages of using user distribution information to optimize MCS selection for different layers, compared to MCS selection schemes presented in references [6,7]. Savings of radio spectrum in the order of 72 to $82 \%$ is observed with the proposed method in different channel quality distributions. There is a good probability for users distributed only in high quality and medium quality regions in an MBSFN network (Scenario B), and a considerable amount of spectrum savings is observed for this case.

Table 14: Percentage savings in spectrum with proposed method

\begin{tabular}{|c|c|c|c|}
\hline \multicolumn{1}{|c|}{ Scenario } & $\begin{array}{c}\text { Total frequency spectrum used } \\
\text { without proposed method (MHz) }\end{array}$ & $\begin{array}{c}\text { Total frequency spectrum used } \\
\text { with proposed method (MHz) }\end{array}$ & $\begin{array}{c}\text { Savings in radio } \\
\text { spectrum }\end{array}$ \\
\hline Scenario A & 14.58 & 14.58 & - \\
\hline Scenario B & 14.58 & 3.96 & $72.83 \%$ \\
\hline Scenario C & 14.58 & 2.52 & $82.71 \%$ \\
\hline
\end{tabular}

\section{Conclusion}

In this paper, we have proposed adaptation and scheduling schemes for both unicast and multicast video transmission over LTE networks using SVC. Narrow band CQI is used for frequency scheduling at eNodeB and wideband CQI is used in the video server for bit rate adaptation in our proposed scheme for unicast. The probability of users being in medium and high channel quality regions are more in MBSFN networks. Hence, we have proposed an adaptive scheme where the MCS of each SVC video layer is chosen based on the channel quality and user distribution for reducing the spectrum usage. The simulation results indicate the advantages of proposed schemes over video transmission without these adaptations. Packet loss ratio is decreased and objective video quality is increased for all the users in cell coverage area for the unicast scheme. Increase of video quality for users in cell edge is also highly noticeable. Furthermore, adaptive GBR selection scheme based on CQI of individual users increase the coverage of the cell considerably. Savings of radio spectrum in the order of 72 to $82 \%$ is observed in different user distribution scenarios with the proposed method for multicast over MBSFN networks. This spectrum can be used for serving other MBSFN, single cell MBMS or unicast bearers or can be used for increasing the video quality of same MBSFN bearer. Future work is planned to improve the current scheme by considering the user mobility without adding much overhead to the existing system. Moreover, MBSFN and other advanced features of LTE make attractive options for optimizing the bandwidth utilization and providing enhanced QoS to cellular users. Further work is also planned in the direction of studying cross layer design options possible in LTE-Advanced networks. 


\section{References}

[1] http://www.itu.int/net/pressoffice/press_releases/2010/40.aspx

[2] J.Huusko, J. Vehkapera, P. Amon, C. Lamy-Bergot, G. Panza, J. Peltola, M.G. Martini, "Cross-layer architecture for scalable video transmission in wireless network", Sig. Proc.: Image Comm. 22(3): 317-330, 2007. http://dx.doi.org/10.1016/j.image.2006.12.011

[3] Esa Piri, Mikko Uitto, Janne Vehkaperä, Tiia Sutinen, "Dynamic Cross-Layer Adaptation of Scalable Video in Wireless Networking”, IEEE Globecom 2010. http://dx.doi.org/10.1109/GLOCOM.2010.5683143

[4] Juniper Research, "Total Revenues from Mobile Entertainment, Regional Forecast 20082012", 2008

[5] "Technical specification group radio access network; Evolved universal terrestrial radio access (E-UTRA) and evolved universal terrestrial radio access network (E-UTRAN); Overall description; Stage 2 (Release 9)", 3GPP TS 36.300, V9.8.0, 2011

[6] A. Correia, N. Souto, A. Soares, R. Dinis, J. Silva, "Multiresolution with Hierarchical Modulations for Long Term Evolution of UMTS," EURASIP Journal on Wireless Communications and Networking, 2009. http://dx.doi.org/10.1155/2009/240140

[7] I-S. Hwang, B-J. Hwang, R-R Su, "Maximizing Downlink Bandwidth Allocation Method Based on SVC in Mobile WiMAX Networks for Generic Broadband Services", ISRN Communications and Networking, 2011. http://dx.doi.org/10.5402/2011/672195

[8] OPNET LTE Specialized Model: http://www.opnet.com/LTE/

[9] Ozgur Oyman, Jeffrey Foerster, Yong-joo Tcha, Seong-Choon Lee, "Toward Enhanced Mobile Video Services over WiMAX and LTE”, IEEE Communication Magazine, 48 (8): 68-76, 2010. http://dx.doi.org/10.1109/MCOM.2010.5534589

[10] T. Wiegand, G. J. Sullivan, G. Bjontegaard, A. Luthra, "Overview of the H.264/AVC video coding standard," IEEE Transactions on Circuits Systems for Video Technology, 13 (7): 560576, 2003. http://dx.doi.org/10.1109/TCSVT.2003.815165

[11] "Improved video support for Packet Switched Streaming (PSS) and Multimedia Broadcast/Multicast Service (MBMS) Services", 3GPP TS 26.903, Mar. 2010

[12] T.Schierl, T.Stockhammer, T. Wiegand, "Mobile Video Transmission Using Scalable Video Coding”, IEEE Trans. On Circuits and Systems for Video Technology, 17 (9): 1204-1217, 2007. http://dx.doi.org/10.1109/TCSVT.2007.905528

[13] H. Juan, H. Huang, C. Huang, T. Chiang, "Cross-layer System Designs for Scalable Video Streaming Over Mobile WiMAX”, IEEE Wireless Communications and Networking Conf., 2007. http://dx.doi.org/10.1109/WCNC.2007.349

[14] K. Järvinen, I. Bouazizi, L. Laaksonen, P. Ojala, A. Rämö, "Media coding for the next generation mobile system LTE", Journal of Computer Communications, 33 (16):1916-1927, 2010. http://dx.doi.org/10.1016/j.comcom.2010.04.019

[15] A. Eichhorn, P. Ni, "Pick your Layers wisely - A Quality Assessment of H.264 Scalable Video Coding for Mobile Devices", IEEE Int. Conf. on Communications, 2009. http://dx.doi.org/10.1109/ICC.2009.5305948

[16] J. C. Ikuno, M. Wrulich, M. Rupp, "System Level Simulation of LTE Networks", IEEE Vehicular Technology $\quad$ Conference, $\quad$ pp. $\quad 1-5, \quad$ May 2010. http://dx.doi.org/10.1109/VETECS.2010.5494007

[17] X. Wang, Y. Zhang, "Optimal video stream multiplexing in MBSFN", IEEE Int. Conf. on Communications Technology and Applications, pp.360-365, Oct. 2009. 
http://dx.doi.org/10.1109/ICCOMTA.2009.5349179

[18] P. Eusebio, A. Correia, "Two QoS Regions Packet Scheduling for MBMS", 2nd Int. Sym. on Wireless Communication Systems, pp. 777-781, Sept. 2005. http://dx.doi.org/10.1109/ISWCS.2005.1547814

[19] A. Alexiou, C. Bouras, V. Kokkinos, A. Papazois, G. Tsichritzis, "Spectral efficiency performance of MBSFN-enabled LTE networks", IEEE Int. Conf. on Wireless and Mobile Computing, pp.361-367, 2010. http://dx.doi.org/10.1109/WIMOB.2010.5645042

[20] K. Etemad, L. Wang, "Multicast and broadcast multimedia services in mobile WiMax networks", IEEE Communications Magazine, 47(10):84-91, 2009. http://dx.doi.org/10.1109/MCOM.2009.5273813

[21] Video Trace Library: http://trace.eas.asu.edu/tracemain.html

[22] J.R. Ohm, "Multimedia Communication Technology", Springer, USA, 2004

[23] C.-H. Lin, C.-H. Ke, C.-K. Shieh, N. K. Chilamkurti, "The Packet Loss Effect on MPEG Video Transmission in Wireless Networks", in Proc. of the 20th Int. Conf. on Advanced Information Networking and Applications (AINA'06), vol. 1, pp. 565 - 572, 2006. http://dx.doi.org/10.1109/AINA.2006.325

[24] JSVM SVC reference software, http://ip.hhi.de/imagecom_G1/savce/downloads/SVCReference-Software.htm, Accessed on Sept. 2011

[25] SVC test sequences, ftp://ftp.tnt.uni-hannover.de/pub/svc/testsequences, Accessed on Oct. 2011

[26] R. Radhakrishnan, A. Nayak, "Cross Layer Design for Efficient Video Streaming over LTE Using Scalable Video Coding", IEEE ICC Workshop on Smart Communication Protocols and Algorithms, Ottawa, Canada, June 2012.

\section{Copyright Disclaimer}

Copyright reserved by the author(s).

This article is an open-access article distributed under the terms and conditions of the Creative Commons Attribution license (http://creativecommons.org/licenses/by/3.0/). 